Musées, Patrimoine et Culture scientifiques et techniques

$192 \mid 2020$

novembre-décembre 2020

\title{
Les matériaux de la conservation préventive
}

\section{Ariane Segelstein}

\section{OpenEdition \\ Journals}

Édition électronique

URL : https://journals.openedition.org/ocim/4168

DOI : $10.4000 /$ ocim.4168

ISSN : 2108-646X

Éditeur

OCIM

Édition imprimée

Date de publication : 1 novembre 2020

Pagination : 22-27

ISSN : 0994-1908

Référence électronique

Ariane Segelstein, «Les matériaux de la conservation préventive », La Lettre de I'OCIM [En ligne], 192 |

2020, mis en ligne le 01 novembre 2021, consulté le 12 décembre 2021. URL : http://

journals.openedition.org/ocim/4168; DOI : https://doi.org/10.4000/ocim.4168

Ce document a été généré automatiquement le 12 décembre 2021.

Tous droits réservés 


\section{Les matériaux de la conservation préventive}

\section{Ariane Segelstein}

Mousses, papier de soie, bacs ou caisses, quels sont les bons choix?

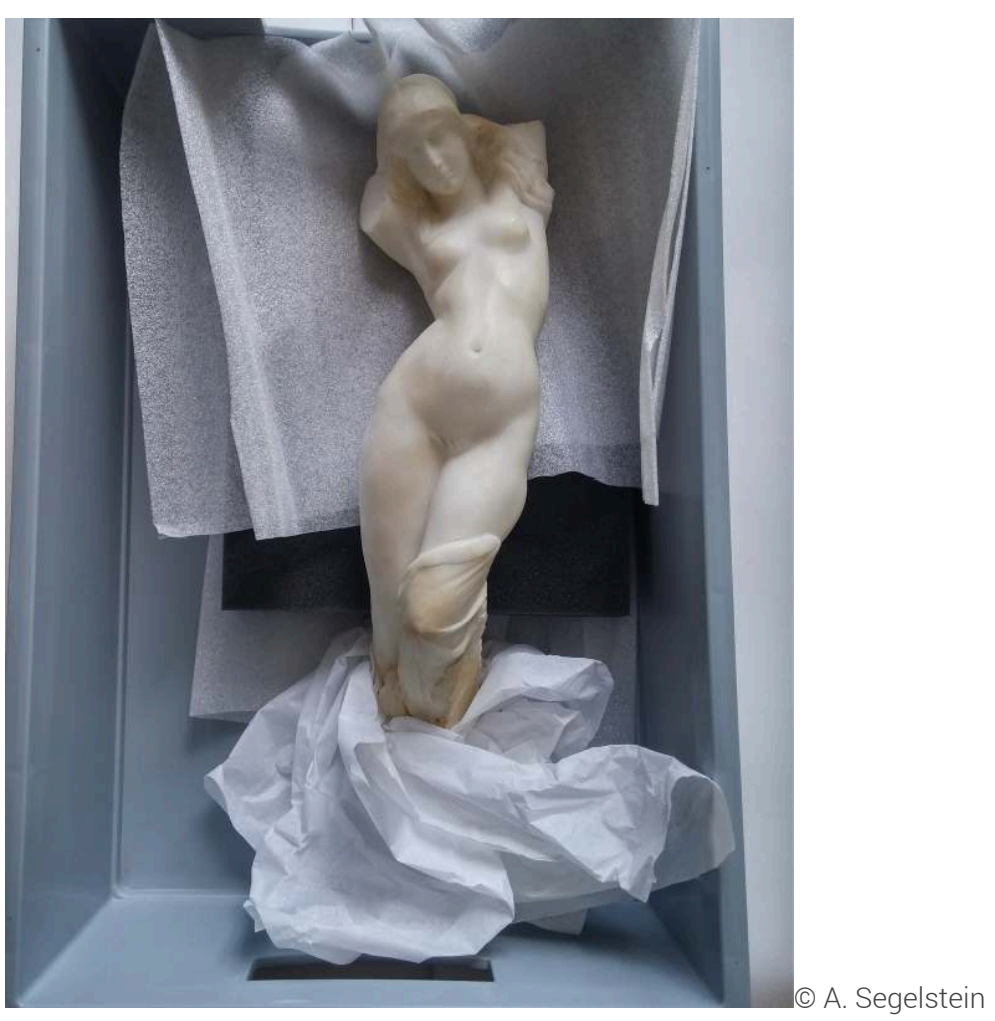

1 Les matériaux de la conservation préventive sont utilisés pour le stockage, le transport et l'exposition des biens culturels. On trouve généralement des matériaux neufs ou usagés sur les lieux même de conservation des collections ou à proximité. Il est donc 
important que chacun puisse se faire une idée du panel de matériaux utilisables et des limites de leurs emplois.

\section{Conditions d'utilisation}

2 Bien connaître une collection permet de la conserver au mieux. Chaque bien culturel a ses caractéristiques et présente des sensibilités qu'il est bon de déterminer. Pour cela un conservateur-restaurateur de biens culturels pourra émettre des conseils avisés.

3 On choisira - quand on le pourra - des matériaux de conservation neufs ou en bon état, qui ne transmettent pas de produits de dégradation aux collections. Ces matériaux doivent être conservés à l'abri de la lumière, dans des espaces propres, ventilés et secs. Il est important de veiller à leur bon état et de les renouveler, car les matériaux de conservation peuvent s'user ou perdre leurs propriétés mécaniques et chimiques. Pour assembler ces matériaux entre eux ou sur eux-mêmes, il est préférable d'utiliser des systèmes d'attache mécaniques plutôt que des systèmes chimiques.

Enfin choisir un matériau pour la conservation, c'est toujours faire un compromis qui tienne compte du contexte, de l'usage, de sa durée, du prix des matériaux ainsi que des propriétés physiques et chimiques que l'on en attend. Les informations qui suivent sont données à titre indicatif, elles ne sauraient être exhaustives. Il est conseillé de se renseigner auprès des fabricants, des fournisseurs et des institutions dédiées à la conservation telles que les laboratoires de recherche, avant de mettre en œuvre des matériaux pour la conservation des biens culturels.

\section{Des risques physiques et chimiques}

5 Choisir un matériau qui soit compatible avec un bien patrimonial nécessite une connaissance des risques liés à l'utilisation de ce matériau. Le choix d'un matériau doit en effet tenir compte de sa compatibilité physique et chimique avec les œuvres: une mousse doit être en adéquation avec le poids d'une œuvre, les surfaces des biens doivent être protégées des abrasions, les contenants doivent être adaptés aux liasses d'archives et non l'inverse, etc.

Il est important de comprendre les mécanismes de pollution et les mouvements des composés nocifs dans les espaces de conservation. Il existe de nombreuses interactions et réactions chimiques possibles entre les composés constitutifs des matériaux de conservation et les matériaux présents dans les bâtiments et les biens culturels. Les composés nocifs pour les œuvres peuvent interagir par contact ou par voie aérienne. Des matériaux inadaptés peuvent participer à la destruction des œuvres et ce de façon parfois très discrète, voire invisible à l'œil nu. Ils peuvent accélérer le vieillissement, provoquer des taches, des décolorations, des déformations, des fendillements, des décompositions partielles et des oxydations. Il est donc préférable d'anticiper les risques et de choisir des matériaux réputés stables, en bon état, que l'on renouvelle régulièrement et de surveiller les œuvres et leurs conditionnements.

Assemblages de matériaux de conditionnement 
Systèmes mécaniques : fil de nylon, aiguille et fil de lin, rivets, ruban à scratch, aiguilles d'entomologiste mais aussi soudage des mousses entre elles, nœuds et autres rubans permettent d'éviter l'ajout d'adhésifs.

Systèmes chimiques : colles, rubans adhésifs, joints.

Systèmes mécaniques d'assemblages de matériaux de conditionnement.

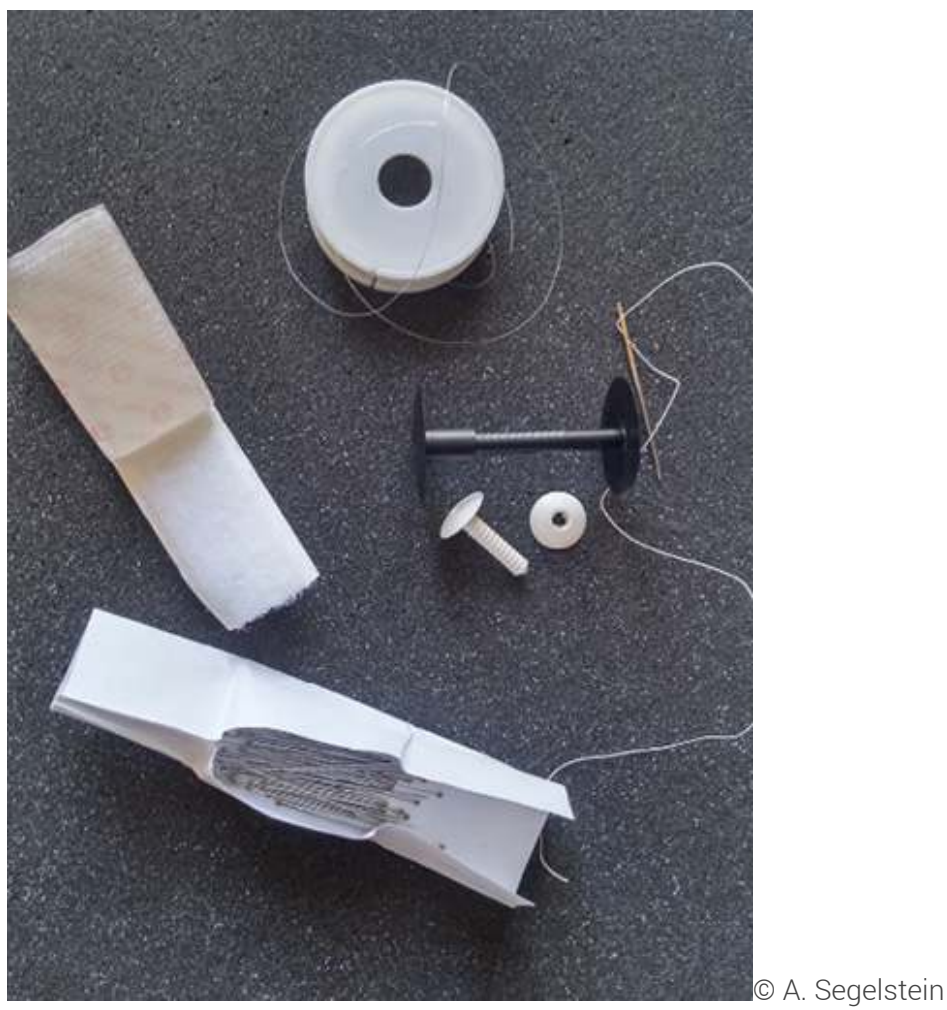

\section{Les matériaux par types}

\section{Les bois}

7 Le bois est un matériau hygroscopique qui absorbe et rejette de la vapeur d'eau en fonction des variations de l'humidité présente dans l'air ambiant. Il agit de ce fait en régulateur qui atténue les variations hygrométriques d'un espace fermé mais les bois libèrent des acides et des tanins. Il est donc déconseillé de mettre les objets en contact direct avec le bois ou dans un milieu fermé. Sont particulièrement sensibles à ces émanations : les métaux; les calcaires; les pigments; les photographies; les textiles; les papiers. Le choix d'un bois pour la conservation s'orientera vers un bois le plus vieux et sec possible et à l'essence peu acide. On le préfèrera sans nœuds et sans traitement fongicide ou ignifuge.

8 Les panneaux dérivés du bois sont très répandus, légers, disponibles dans de grandes dimensions et peu coûteux. Ce sont les contre-plaqués, contre-plaqués lattés, contreplaqués lamellés, les panneaux de particules, les panneaux de fibres dont le fameux médium. Les panneaux d'essences de bois différentes et d'adhésifs aux formaldéhydes (en dehors des phénol) sont un danger potentiel pour les collections. Il est déconseillé 
de mettre des objets dans leur environnement ou à leur contact. Certains panneaux de bois sont de plus très sensibles à l'humidité. Celle-ci provoque des gonflements, des délitages des particules constitutives, des émanations nocives supplémentaires. On notera qu'en plus des acides, les mauvais adhésifs libèrent leurs propres produits de dégradation. Si la structure des panneaux est trop fragile, les placages peuvent se casser ou se décoller. Pour choisir un panneau de bois on regardera la nature de la résine, les plus sûres sont les mélamines, les phénol formaldéhydes, le polyurée et l'époxy. On veillera aussi à opter pour des panneaux de classe A ou 1 qui correspondent aux taux de formaldéhydes les plus bas. Bois et panneaux de bois qui émettent des composés nocifs peuvent être isolés des œuvres avec des produits barrières aussi appelés « pare-vapeur ».

\section{Les papiers et les cartons}

9 Le principal critère de qualité d'un papier de conservation est sa faible acidité. L'acidité d'un papier ou d'un carton peut provenir de ses propres constituants ou de composés extérieurs. Un papier fait de pâte de bois est plus ou moins jaune. Pour satisfaire aux exigences commerciales les pâtes sont blanchies avec des produits qui peuvent être des composés chlorés, de la soude, de l'eau oxygénée, de l'eau de soude, des azurants optiques, des agents fluorescents ou des pigments. Ces agents ou leurs résidus peuvent modifier les propriétés des papiers et des cartons.

10 Les papiers et cartons à réserve alcaline contiennent une charge alcaline minérale d'environ $3 \%$ de carbonate de calcium ou de magnésium. Cette charge alcaline atténue l'acidification du papier due à sa propre dégradation ou à la proximité de composants acides. La charge alcaline s'épuise avec le temps, d'autant plus vite que le papier est exposé à un environnement fortement acide.

11 On choisira de préférence les papiers « pur chiffon » (100\% coton, $100 \%$ cellulose), les «pâtes chimiques» de bois très raffinées qui ne contiennent que de la cellulose et qui de ce fait se rapprochent beaucoup des papiers "pur chiffon", les papiers de soie non acides, les papiers d'archives. Les cartons non acides alvéolés, ondulés ou à plis sont aussi de très bons produits. Certains papiers sont à éviter d'une manière générale mais ils peuvent être bien utiles en cas d'urgence ou pour des transports temporaires : ce sont le papier journal sans encre, le papier kraft, le papier de soie ordinaire, les cartons ordinaires.

\section{Les textiles}

Les textiles sont hygroscopiques quand ils sont naturels, ils agissent comme des régulateurs de l'humidité. Ils présentent une vulnérabilité aux micro-organismes et sont des foyers pour les insectes quand ils sont poussiéreux et humides. Pour ces raisons il est conseillé de les dépoussiérer régulièrement et de maintenir les réserves propres. Les textiles peuvent avoir été apprêtés ou contenir des substances ignifuges ou fongicides. Il est donc recommandé, si possible, de les laver avec du savon de Marseille et de bien les rincer avant utilisation. Les textiles pouvant perdre leurs fibres sont à éviter. Un grand avantage de l'utilisation des textiles est la possibilité de les coudre et d'éviter - ou de limiter - l'utilisation d'adhésifs. Les textiles seront préférentiellement de couleurs écrues ou neutres. Si l'on choisit des textiles colorés, il est nécessaire de 
tester leur résistance à la décoloration et leur innocuité pour les collections. Les textiles blancs peuvent avoir été blanchis et contenir des résidus issus de ces opérations. Un choix sûr se portera vers des textiles non pelucheux, tels que le drap de coton; le jersey de coton; le lin; les polyesters; les acryliques et les polyéthylènes. On évitera les textiles protéiques tels que la laine et la soie qui peuvent émettre des composés soufrés.

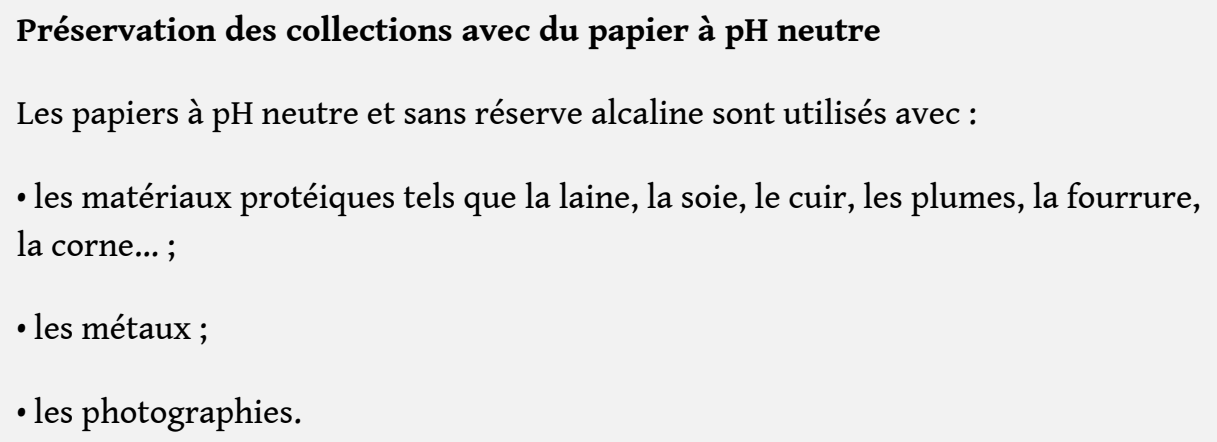

Le cuir et la fourrure peuvent être conservés dans des boites en carton non acide garnies de papier de soie à $\mathrm{pH}$ neutre.

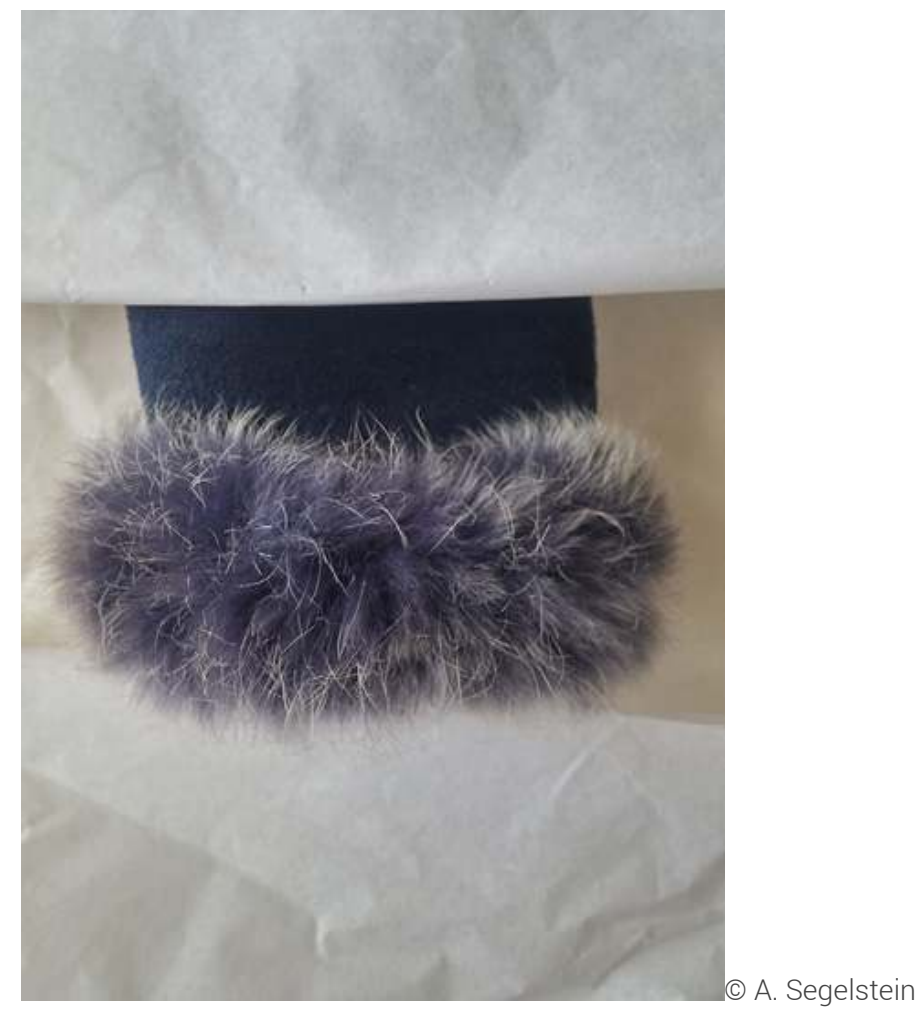

\section{Les polymères synthétiques}

La fabrication des plastiques se fait avec une résine de base qui détermine les caractéristiques du matériau et à laquelle on ajoute souvent des additifs qui modifient leurs propriétés. Les additifs sont généralement présents en faibles quantités mais pour certains, comme les plastifiants, leur taux peut dépasser $60 \%$ en masse. Les plastifications internes qui sont produites par l'assemblage de plusieurs polymères 
pour réaliser un copolymère ne posent pas de problème de stabilité. À l'inverse, les plastifications externes qui fonctionnent par l'adjonction d'un liquide dissout dans le polymère n'assurent pas la stabilité nécessaire à la conservation des collections. Le plastifiant risque de migrer vers la surface et à terme de rendre le plastique poisseux. Les plastiques sont électrostatiques ce qui pose des problèmes pour la conservation des biens pulvérulents, mais aussi ceux dont la cohésion matérielle est sensible. Des traitements antistatiques existent mais ces additifs incorporés aux plastiques par un intermédiaire aqueux rendent les polymères plus hygroscopiques et sensibles à l'hydrolyse. Ils sont donc peu adaptés à la conservation des biens culturels. Les ralentisseurs de flamme sont nocifs au contact pour les biens culturels, notamment pour les métaux. Les plastiques instables peuvent altérer les biens environnants par le dégagement de composés organiques volatils ou non. Un bon choix consiste à sélectionner les plastiques noirs, blancs, gris ou incolores qui contiennent le moins d'additifs possible.

14 Les résines les plus sûres sont les polyéthylènes, les polyesters, les polypropylènes, les polystyrènes si on ne les met pas en contact avec les métaux, l'acrylonitrile-butadiènestyrène, le polytetrafluoroéthylène, les polycarbonates, les polyamides, les acryliques.

Mousses, films, plaques, fibres de plastique utilisés pour la conservation des collections.

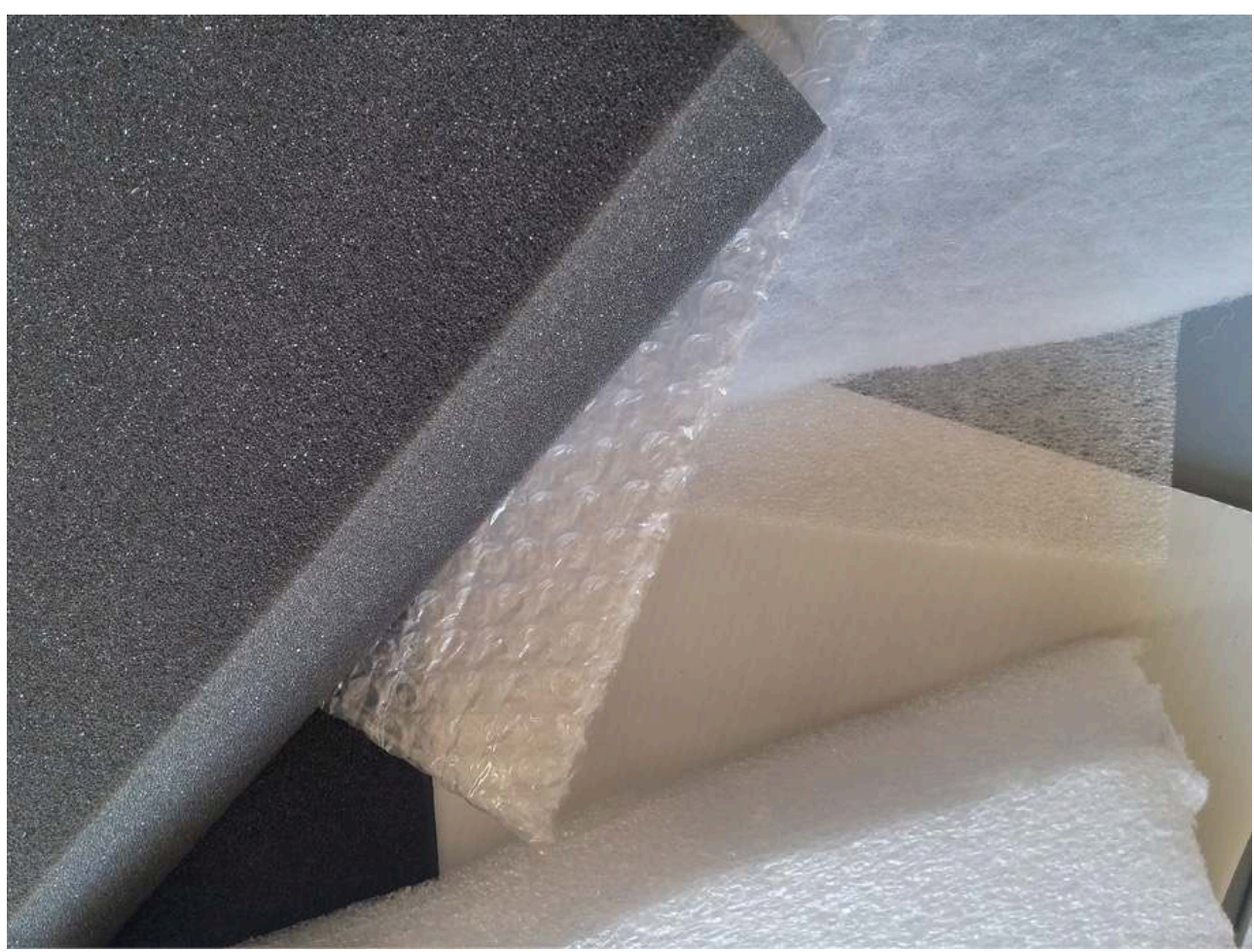

(c) A. Segelstein

\section{Les produits barrières}

Il est parfois nécessaire d'isoler un bien d'un matériau susceptible de l'endommager par des émanations. Il peut s'agir d'isoler un bois, un panneau de bois, des plastiques, des mousses, des peintures ou des vernis. Les produits barrières ou pare-vapeur sont utiles dans des caisses de transport, sur des étagères ou dans des vitrines d'exposition. Les 
espaces confinés seront conçus en tenant compte de la vulnérabilité des biens et avec des matériaux les plus inertes possible, chimiquement stables et bien secs. On distingue les produits barrières passifs des produits barrières actifs.

Les produits dits passifs sont des peintures, des vernis, des films complexes d'aluminium et de plastiques, des feuilles de plastique. Les peintures et les vernis seront choisis de façon à limiter autant que possible les composés organiques volatils et seront séchés dans un espace ventilé pendant au moins quatre semaines avant leur utilisation dans l'environnement des biens culturels.

Les produits dits actifs sont des composés filtrants au cuivre, aux charbons actifs ou aux zéolithes. Ce sont des papiers et des cartons, des textiles, des peintures ou des films plastiques qui emprisonnent des molécules d'eau et de polluants. Il faut savoir que ces substances adsorbantes finissent par se saturer et peuvent rejeter les polluants accumulés. Il est donc nécessaire de les renouveler régulièrement et de veiller aux taux d'humidité ambiants.

Les films barrières, complexes de polyéthylène et d'aluminium empêchent le passage des vapeurs nocives émises par les bois et les panneaux dérivés du bois.

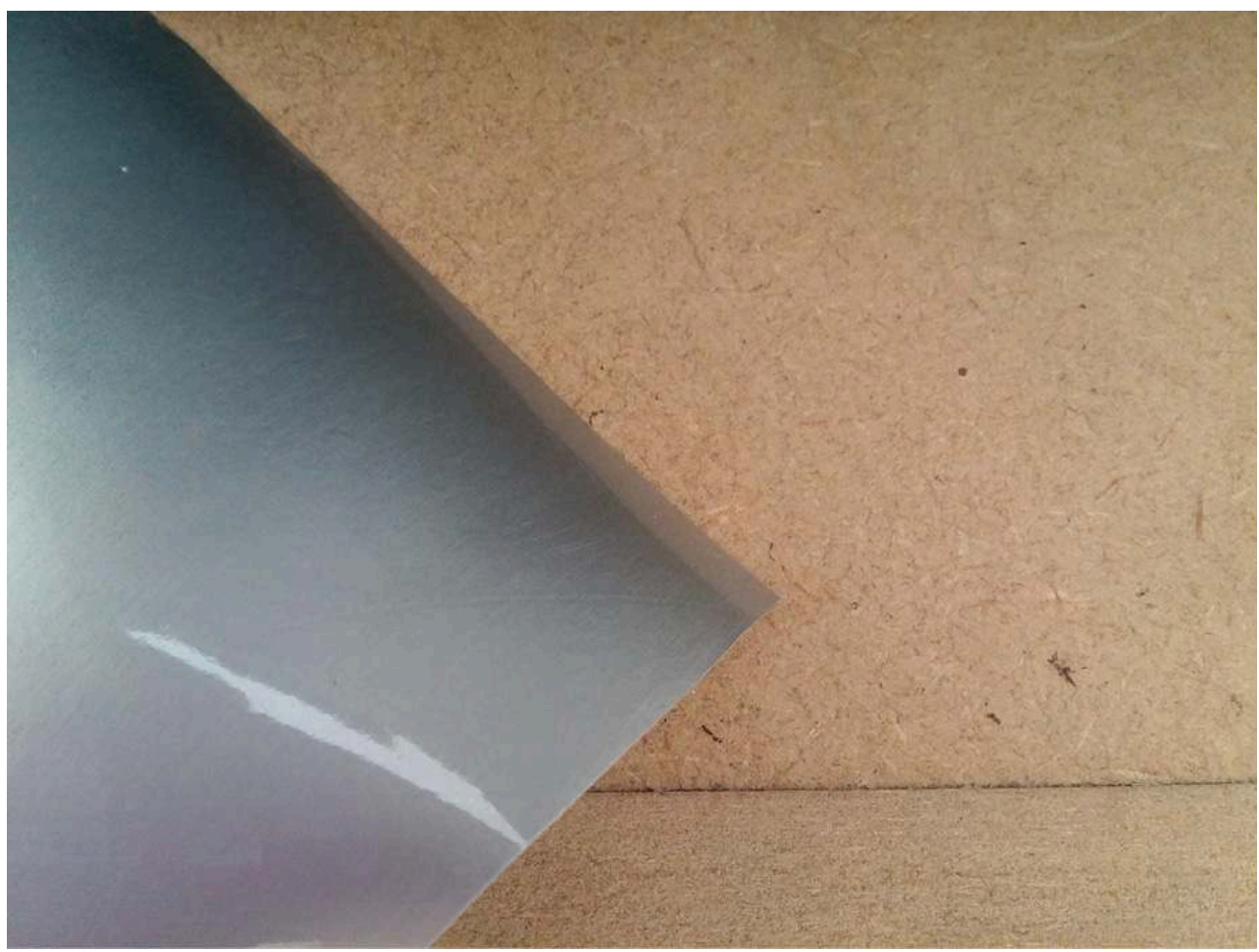

(C) A. Segelstein

\section{Conclusion}

18 La proximité des matériaux avec les biens patrimoniaux avec lesquels ils sont en contact direct et souvent pour de longues périodes, nécessite que l'on considère les risques avec attention. Choisir les matériaux que l'on utilise dans l'environnement des collections devrait toujours se faire en connaissance de cause du fait de leurs possibles effets sur les collections. 

œuvre est également très importante pour la bonne conservation des biens à préserver, y compris lors des transports et des expositions.

\section{BIBLIOGRAPHIE}

Goffard C. Éviter l'erreur : le choix de matériaux stables pour le stockage et l'exposition des collections muséales, CeROArt, n³, 2009,

[en ligne].

Nguyen T. Analyse des matériaux et normes, Actualités de la conservation, n¹7, mai-août 2002, pp. 7-8.

Afnor Normalisation. Conservation des biens culturels: Principes d'emballage pour le transport. Norme NF EN 15946, La Plaine Saint Denis : Afnor, octobre 2011, 22 p.

Afnor Normalisation. Prescriptions et critères de sélection des papiers et cartons pour la conservation des documents papiers et parchemins. Norme NF Z40-014, Afnor, mai 2011, 9 p.

Segelstein A. Prototype d'une base de données sur les produits utilisés en conservation préventive pour le stockage, l'exposition et le transport des collections, Technè, n8, 1998, pp. 81-86.

Tétreault J. Matériaux de construction, matériaux de destruction. In La conservation préventive, ARAAFU, Paris, 1992, pp. 163-176

Tétreault J. Display materials : the good, the bad and the ugly. In Sage J. Exhibitions and Conservation: Pre-prints of the Conference held at The Royal College of Physicans (Édimbourg, 21-22 avril 1994). Édimbourg (Écosse) : The Scottish Society for Conservation \& Restoration (SSCR), 1994, pp. 79-87, [en ligne].

\section{RÉSUMÉS}

Les matériaux utilisés pour la conservation préventive des collections possèdent chacun des qualités précises qu'il convient de connaitre pour protéger au mieux les objets avec lesquels ils pourraient être en contact. Une spécialiste de la conversation-restauration revient sur les principaux risques inhérents à leur utilisation.

\section{INDEX}

Mots-clés : conservation préventive, matériau (conservation) 
AUTEUR

\section{ARIANE SEGELSTEIN}

Conservatrice-restauratrice de sculpture contemporaine, consultante en conservation préventive et formatrice, diplômée de l'université de Paris 1 Panthéon-Sorbonne as@preservationdescollections.com 